visiting hours. The common medical problems listed in the patient log were: (1) respiratory diseases: $40 \%$; (2) skin disorders: $15 \%$; (3) orthopedic disorders and myalgia: $15 \%$; (4) asthma: $10 \%$; (5) anxiety and insomnia: $5 \%$; and (6) wound evaluation and dressing changes: $5 \%$. Many patients visited the clinic without any concrete medical complaints in order to talk to the staff and receive emotional support.

Conclusion: The primary healthcare needs within the evacuation center for disaster victims were relatively large. The evacuation center and the clinic became operational on the day following the tsunami. Most recorded visits were for medical problems not directly related to the actual natural disaster, with respiratory diseases including asthma accounting for $50 \%$ of all visits. The rapid deployment of a health clinic staffed by medical personnel from unaffected areas of the country and volunteers from abroad enabled coping with the medical needs of the evacuation center population.

Keywords: clinics; diseases; emotional support; primary health care; staffing; tsunami; visits

Prehosp Disast Med 2005;20(3):s148-s149

\section{Thailand's Medical System Response to the Tsunami Disaster: Infrastructure, Population and Medical Teams}

Y. Bar-Dayan; ${ }^{1}$ A. Leiba; ${ }^{1}$ I. Ashkenasi; ${ }^{2}$ G. Nakash; ${ }^{1}$

R. Pelts, ${ }^{1}$ D. Shvarts, ${ }^{3}$ A. Goldberg, ${ }^{3}$ Y. Levi ${ }^{2}$

1. IDF Home Front Command, Israel

2. IDF Medical Corps, Surgeon General Headquarters, Israel

3. Faculty of Health Sciences, Ben Gurion University, Israel

Introduction: On 26 December 2004, an earthquake with a magnitude of 9.0 on the Richter scale struck the area off the western coast of northern Sumatra, triggering massive tidal waves (tsunami). The tsunami waves inundated costal areas in countries all around the Indian Ocean rim including Thailand, causing destruction of infrastructure and a huge number of fatalities and injuries. On 25 January 2005 in Thailand, 5,388 fatal cases were confirmed, 3,120 people were reported missing, and 8,457 patients were wounded. Key elements of the medical system response in Thailand were analyzed in order to delineate the unique characteristics of such a disaster and the preferred mode of preparedness.

Methods: The IDF home front command medical department sent a research delegation to study the response of the medical system in Thailand to the disaster. The delegation included three senior military physicians and a population behavior sociologist who are experienced in hospital preparedness to disaster and emergency medicine. The delegation worked from 29 January-04 February and questioned Generals of the Thai Air Force, the Director of the Ministry of Health, three provincial health services directors, six managers of public hospitals, two heads of community clinics, and two rescue camps community health centers' commanders and their teams. The collection of data was done by open and closed questionnaires, reading the debriefing reports of each organization, and observation of the infrastructure of all of the medical facilities.

Results: There was no alert before the event occurred. In the first minutes after the event, survivors reported their life-saving behaviors: climbing trees, running to the mountains, or giving aid to their beloved. Interestingly, an exceptional survival of children in some villages were found, where children were trained, as part of a popular children's game, to run from the sea waves to the mountains.

First responders were mostly the injured survivors themselves and their relatives, who arrived to hospitals in any way they could. On Phi Phi Island, there were 700 casualties for every physician. For that reason, these few physicians reported being busy in patients' triage and hemmorhage control. For the first day after the tsunami, there was effectively no communication between Phi Phi Island and Thailand. Hospitals in the affected provinces were overwhelmed by casualties - between 200 and 1,100 casualties in one hospital. Secondary distribution was done from the affected area's hospitals to hundreds of medical facilities and hospitals all over the country. Medical response was based on upgrading and reinforcement of the regular medical facilities in Thailand and not on the rapid deployment of new ones. Hospitals' crews who reported having participated in a recent drill or any other preparedness plan reported a better personal capability to give medical aid, compared to those who were not prepared.

Discussion: A well-organized alarm and communication system could save many lives. An operational alarm system, similar to war-time alarm, arriving one and one-half hours before the wave flooded Thailand's shores, could have saved many lives. An efficient and well-structured communication system could enable a faster recruitment of medical forces to Phi Phi Island, as well as faster evacuation from the island to hospitals in Thailand.

Population preparedness should focus on ongoing teaching of first aid and training of rescue maneuvers. Preparing children using games and simulations can be less frightening, but still effective. Education of first responders should concentrate on rapid triage and hemmorhage control.

Overwhelmed hospitals should rearrange themselves for triage and life-saving treatment followed either by discharge or secondary distribution to many other medical facilities. In such a way, every hospital deals with fewer casualties and can provide a reasonable level of medical care. The model of reinforcement and versatility of the existing medical facilities seems to be applicable during natural disasters, especially in a scenario like this, in which there is lack of communication. Casualties, who evacuate themselves in such a chaotic scenario, would find it easiest to arrive at the near-by medical facilities that serve them everyday. Preparedness of hospitals' crews is crucial. Prepared personnel felt much more suited, both medically and mentally, to deal with a disaster of this scale.

Conclusion: Preparedness for a tsunami is very similar to preparedness for wartime scenario. It should include three main elements: (1) preparing infrastructure and alert systems; (2) preparing the population of all ages; and (3) preparing hospitals' crews.

Keywords: alert; children; communication; games; hospitals; infrastructure; preparedness; tsunami

Prebosp Disast Med 2005;20(3):149 